Research article

\title{
Wheat bran valorisation: Towards photocatalytic nanomaterials for benzyl alcohol photo-oxidation
}

\author{
Weiyi Ouyang a, Jose M. Reina a, Ewelina Kuna b, Alfonso Yepez a , Alina M. Balu a, \\ Antonio A. Romero ${ }^{\text {a }}$ Juan Carlos Colmenares ${ }^{b}$, Rafael Luque ${ }^{a, *}$ \\ a Departamento de Quimica Organica, Universidad de Cordoba, Edificio Marie Curie(C-3), Ctra Nnal IV-A, Km 396, E14014 Córdoba, Spain \\ ${ }^{\mathrm{b}}$ Institute of Physical Chemistry PAS, Kasprzaka 44/52, 01-224 Warsaw, Poland
}

\section{A R T I C L E I N F O}

\section{Article history:}

Received 3 February 2016

Received in revised form

3 July 2016

Accepted 4 July 2016

Available online $\mathrm{xxx}$

\section{Keywords:}

Waste valorization

Wheat bran

Photocatalysis

Benzyl alcohol oxidation

\begin{abstract}
A B S T R A C T
In this work, we have successfully synthesized a set of titania photocatalytic nanocomposites by the incorporation of different $\mathrm{TiO}_{2}$ content on wheat bran residues. The obtained catalysts were characterized by different techniques including UV-Vis spectroscopy, X-ray diffraction (XRD), X-ray photoelectron spectroscopy (XPS) and Transmission Electron Microscopy (TEM) while their photocatalytic activity was investigated in the oxidation of benzyl alcohol under UV light irradiation. Benzaldehyde yields were ca. $20 \%$, with conversion in the systems of ca. $33 \%$ of benzyl alcohol by using $10 \%$ Ti-Bran catalyst, as compared to $33 \%$ yield to the target product (quantitative conversion of benzyl alcohol) using commercial pure $\mathrm{TiO}_{2}$ (P-25). The photocatalytic activity results indicate that designed waste-derived nanomaterials with low $\mathrm{TiO}_{2}$ content can efficiently photocatalyze the conversion of benzyl alcohol with relative high selectivity towards benzaldehyde.
\end{abstract}

() 2016 Elsevier Ltd. All rights reserved.

\section{Introduction}

Environmentally friendly and energy-saving technologies are becoming increasingly advantageous with the increase of public awareness on environment protection and sustainable development. Since the discovery of photocatalytic splitting of water on $\mathrm{TiO}_{2}$ electrodes (Fujishima and Honda, 1972), semiconductor-based heterogeneous photocatalysis has received extensive attention and now is considered to be one of the most relevant and environmentally friendly technologies with advantages including simplicity, environmental compatibility, low-cost and energysaving.

To assess the photocatalytic activity of a semiconductor photocatalyst, several factors should be taken into consideration, including its stability, efficiency, selectivity and wavelength range response (Linsebigler et al., 1995). Among various semiconductorbased photocatalysts, $\mathrm{TiO}_{2}$ is one of the most attractive photocatalysts owing to its outstanding photocatalytic activity, high thermal and chemical stability, non-toxicity, cost effectiveness and the strong oxidizing power of the photogenerated holes. Massive

\footnotetext{
* Corresponding author.

E-mail address: q62alsor@uco.es (R. Luque).
}

investigations related to $\mathrm{TiO}_{2}$ photocatalysts have been reported in different applications in the last decades, including chemicals and fuels production from $\mathrm{CO}_{2}$ reduction (Adachi et al., 1994; Chen et al., 2015; Yu et al., 2014), decontamination of water (Herrmann et al., 1999; Hoffmann et al., 1995; Virkutyte et al., 2010; Yang et al., 2008), organic synthesis (Colmenares et al., 2016; Higashimoto et al., 2009; Hubert et al., 2010; Ohno et al., 2003b) and production of $\mathrm{H}_{2}$ from water splitting (Chen et al., 2010; Ni et al., 2007). However, the use of pure $\mathrm{TiO}_{2}$ photocatalyst is limited by its large band gap (3.2eV, anatase crystalline phase) which requires light with wavelength $\lambda<387 \mathrm{~nm}$ for the excitation of electrons from the valence to the conduction band, resulting that only $5 \%$ of the solar irradiation can be utilized for the photocatalytic process. Modifications on $\mathrm{TiO}_{2}$ are necessary to improve its photocatalytic properties under solar irradiation or artificial light source. Modifications such as introductions of metals, dopants or combinations with other semiconductors are beneficial because they suppress the recombination of the photogenerated electron-hole pairs and thus increase the quantum yield of the photocatalytic process (Linsebigler et al., 1995). Meanwhile, the newly created heterojunctions can help the photocatalyst expand the wavelength range response into visible light region.

Many reported investigations have revealed that doping with non-metal atoms, such as C, N, F, P or S, can significantly improve 
the photocatalytic activity of $\mathrm{TiO}_{2}$ under visible light (Chang et al., 2005; Irie et al., 2003; Ohno et al., 2004, 2003a; Sun et al., 2008; Virkutyte and Varma, 2010; Virkutyte et al., 2010; Wu et al., 2007). Co-doping of $C$ and $N$ (Chen et al., 2007; Yang et al., 2008), metal and non-metal co-doping (Neville et al., 2012; Shen et al., 2008; Virkutyte and Varma, 2010) were reported to render materials with high visible light response.

Selective oxidation of benzyl alcohol to benzaldehyde is an attractive research topic since benzaldehyde is extensively used in the food, perfumery and pharmaceutical industries, as well as precursor in other chemical industries and present in a wide range of commercial formulations. Moreover, the study of the conversion of benzyl alcohol to benzaldehyde can potentially provide basic reactivity and transformation strategies for the valorization of lignocellulose-based biomass (e.g. lignin). Photocatalytic oxidation of benzyl alcohol towards benzaldehyde with high selectivity has been reported in recent years (Colmenares et al., 2016; Higashimoto et al., 2009; Li et al., 2012; Marotta et al., 2011; Spasiano et al., 2013), which provide a possible substitution to the traditional synthetic routes - either by benzyl chloride hydrolysis or through oxidation of toluene. A review on the transformation of biomass-derived compounds with heterogeneous photocatalytic nanomaterials was also recently reported (Colmenares and Luque, 2014).

In the present investigation, we have developed a novel, low cost $\mathrm{TiO}_{2}$ photocatalytic nanocomposite (denoted as Ti-Bran) derived from the valorization of a widespread and highly abundant industrial waste such as wheat bran. Catalysts with different $\mathrm{TiO}_{2}$ content on the wheat bran support were synthesized. The photocatalytic activity was tested by the oxidation of benzyl alcohol to benzaldehyde. Different techniques were applied in the characterization of the catalysts including Nitrogen physisorption, X-ray diffraction (XRD), X-ray photoelectron spectroscopy (XPS), Transmission Electron Microscopy (TEM) and UV-Vis spectroscopy.

\section{Material and methods}

Titanium isopropoxide (IV), Sigma-Aldrich 99\%, Wheat bran was kindly donated by a local company as a byproduct of wheat refining for food products. All remaining reagents including benzyl alcohol and acetonitrile (99\%, Sigma-Aldrich) and related others were used as purchased.

\subsection{Catalyst synthesis}

For the catalyst synthesis, several different nanomaterials were prepared an investigated with the same quantity of waste starting material (wheat bran, $5 \mathrm{~g}$ ) and different quantities of titanium isopropoxide as illustrated on Table S1 to reach different theoretical contents of titanium (from 0.5 to $10 \mathrm{wt} \%$ ) in the final material. In a typical synthesis, the respective mixtures containing wheat bran $(5 \mathrm{~g})$ and the different quantities of titanium precursor were milled in a planetary ball mill RETSCH PM 100 model under previously optimized conditions (350 rpm, $30 \mathrm{~min}$ ) (Francavilla et al., 2014). 18 stainless steel balls ( $1 \mathrm{~cm}$ diameter) were utilized in the stainless steel milling chamber. After milling, the final powdery mixture was collected and subjected to calcination at $400{ }^{\circ} \mathrm{C}$ for $5 \mathrm{~h}$ (heating ramp $3{ }^{\circ} \mathrm{C} \mathrm{min}^{-1}$ ) to partially remove the organic matter from the wheat bran, leaving a nice brownish Ti-Bran final product. Only a $6-15 \%$ of the weight of the initial mixture was obtained after calcination, indicating that most of the wheat bran (organic material) was eliminated in the calcination step. The temperature used for calcination was $400{ }^{\circ} \mathrm{C}$ because of the preferential formation of anatase phase at lower temperatures $\left(450{ }^{\circ} \mathrm{C}\right.$ and below) as compared to the formation of a rutile poorly active phase in photocatalysis obtained at higher temperatures (Colmenares and Luque, 2014).

\subsection{Materials characterization}

Nitrogen porosimetry studies were conducted in a Micromeritics ASAP 2000 volumetric adsorption analyzer at 77 K. Samples were degassed at $100{ }^{\circ} \mathrm{C}$ during $24 \mathrm{~h}$ under vacuum $\left(p<10^{-2} \mathrm{~Pa}\right)$ prior to analysis. The linear part of the BET equation (partial pressures in the 0.05 and 0.30 range) was used to determine the specific surface area. The pore size distribution was calculated from the adsorption branch of the $\mathrm{N}_{2}$ physisorption isotherms using the Barret-Joyner-Halenda (BJH) formula. The cumulative mesopore volume $\mathrm{V}_{\mathrm{BJH}}$ was obtained from the PSD curve.

XRD patterns were obtained in a Bruker D8 DISCOVER A25 diffractometer equipped with a vertical goniometer under thetatheta geometry using $\mathrm{Ni}$ filtered $\mathrm{Cu} \mathrm{K} \alpha(\lambda=1,5418 \AA)$ radiation and operated at $40 \mathrm{KeV}$ and $40 \mathrm{~mA}$. Wide angle scanning patterns were collected from 10 to $80^{\circ}$ with a step size of $0.01^{\circ}$ and counting time of 500 s per step.

The light absorption properties of the photocatalysts were investigated through UV-Vis spectroscopy. UV-Vis diffuse reflectance spectra were recorded on UV/VIS/NIR spectrophotometer Jasco V-570 equipped with an integrating sphere. The baseline was recorded using Spectralon ${ }^{\mathrm{TM}}$ (poly(tetrafluoroethylene) as a reference material. The Kubelka-Munk method based on the diffuse reflectance spectra was applied to determine the band gap function. The $E_{g}$ was calculated form $(\mathrm{f}(\mathrm{R}) \mathrm{h} v)^{1 / 2}$ versus $\mathrm{h} v$ plots. The function $f(R)$ was calculated from the following Equation (1):

$f(R)=\frac{(1-R)^{2}}{2 R}$

XPS measurements were conducted at an ultrahigh vacuum (UHV) multipurpose surface analysis system (Specs ${ }^{\mathrm{TM}}$ model, Germany) at the SCAI in Universidad de Cordoba, operating at pressures $<10^{-14} \mathrm{MPa}$ using a conventional X-Ray source (XR-50, Specs, $\mathrm{Mg}-\mathrm{K} \alpha, 1253.6 \mathrm{eV}$ ) in a "stop-and-go" mode to reduce potential damage due to sample irradiation. The survey and detailed highresolution spectra (pass energy 25 and $10 \mathrm{eV}$, step size 1 and $0.1 \mathrm{eV}$, respectively) were recorded at room temperature using a Phoibos 150-MCD energy analyzer. Powdered samples were deposited on a sample holder using double sided adhesive tape and subsequently evacuated under vacuum $\left(<10^{-6}\right.$ Torr) overnight. Eventually, the sample holder containing the degassed sample was transferred to the analysis chamber for XPS studies. Binding energies were referenced to the $\mathrm{C} 1 \mathrm{~s}$ line at $284.6 \mathrm{eV}$ from adventitious carbon.

Transmission Electron Microscopy (TEM) images for selected TiBran nanocomposites were recorded in a FEI Tecnai G2 fitted with a CCD camera for ease and speed of use at the SCAI from Universidad de Cordoba. The resolution is around $0.4 \mathrm{~nm}$. Samples were suspended in ethanol and deposited straight away on a copper grid prior to analysis.

ICP-MS experiments were conducted at the SCAI of Universidad de Cordoba. Prior to analysis and metal quantification, samples were prepared by treating $0.05 \mathrm{~g}$ of nanocomposite material with $1 \mathrm{~mL}$ concentrated HF (till complete dissolution of the powder) in special recipients. In some cases, a few drops of $\mathrm{HNO}_{3}$ and $\mathrm{HCl}$ were also added to ensure complete dissolution of the samples, which were subsequently topped with milli Q water to $25 \mathrm{~mL}$ total volume. Samples were then immediately analysed in an ICP/MS Perkin Elmer ELAN-DRC-e model equipped with an automatic diluting injecting system and ionisation under $\mathrm{Ar}$ plasma followed by quadrupole ion detection with a DRC cell to remove potential 
interferences.

\subsection{Photocatalytic activity experiments}

The photocatalytic activity of the synthesized Ti-Bran catalysts was tested in the oxidation of benzyl alcohol to benzaldehyde. All reactions were performed in a Pyrex cylindrical double-walled immersion well reactor equipped with medium pressure $125 \mathrm{~W}$ mercury lamp $(\lambda=365 \mathrm{~nm})$, supplied by Photochemical Reactors Ltd. UK (Model RQ 3010). A homogeneous suspension of the catalyst was obtained by means of magnetic stirring at $1100 \mathrm{rpm}$. Benzyl alcohol was prepared in acetonitrile medium with an initial concentration of $1.5 \mathrm{mM}$. The reaction temperature was set at $30^{\circ} \mathrm{C}$. Experiments were performed from $150 \mathrm{~mL}$ of the mother solution with catalyst loading of $1 \mathrm{~g} / \mathrm{L}$ under UV light for $4 \mathrm{~h}$. Air bubbling was also applied with a flow rate at $25 \mathrm{~mL} / \mathrm{min}$. The reaction solution was put into dark environment for $30 \mathrm{~min}$ prior to the reaction in order to equilibrate the adsorption-desorption over the photocatalyst surface. At each sampling point, $1 \mathrm{~mL}$ sample was collected directly from the reactor and filtered $(0.2020 \mu \mathrm{m}, 25 \mathrm{~mm}$, nylon filters). High performance liquid chromatography (HPLC, Waters Model 590 pump) equipped with a Dual Absorbance Detector (Waters 2487) and the SunFire ${ }^{\mathrm{TM}}$ C18 (3.5 $\mu \mathrm{m}, 150 \mathrm{~mm}$ length, $4.6 \mathrm{~mm}$ inner diameter) column provided by Waters was applied to determine the concentration of the model compound. The mobile phase was Milli-Q water/acetonitrile/methanol in the volumetric ratio of 77.5: 20: 2.5 with $0.1 \%$ of $\mathrm{H}_{3} \mathrm{PO}_{4}$. The isocratic elution flow rate was $1 \mathrm{~mL} / \mathrm{min}$ and the injection volume was $10 \mu \mathrm{L}$.

\section{Results and discussion}

The synthesized nanocomposites denoted as Ti-Bran were characterized using a number of analytical techniques. Nitrogen physisorption isotherms of Ti-containing nanocomposites indicated the presence of a certain interparticle macro/mesoporosity in the materials after the calcinations process, most probably due to the removal of some organics from the decomposition of wheat bran (Fig. S1). Materials were essentially non porous in nature, as expected from wheat bran, although the observed interparticle porosity was reflected in the observed not negligible surface areas $\left(<20 \mathrm{~m}^{2} \mathrm{~g}^{-1}\right.$, Table 1$)$.

XRD patterns compiled in Fig. 1 pointed to the clear presence of a distinctive anatase phase in the materials, together with the presence of additional crystalline phases (not quantified) of a range of metal oxides in low quantities including $\mathrm{Zn}$, Mn and related trace elements detected in ICP/MS analysis (not quantified). All materials exhibited almost identical XRD patterns differing only in terms of the intensity of the main diffraction lines. The calculated titania nanoparticle size using the Scherrer equation from XRD patterns was of ca. 7-10 nm for all synthesized nanomaterials, slightly increasing at high Ti loadings in the materials (Table 1).

Further XPS characterization studies confirmed the presence of

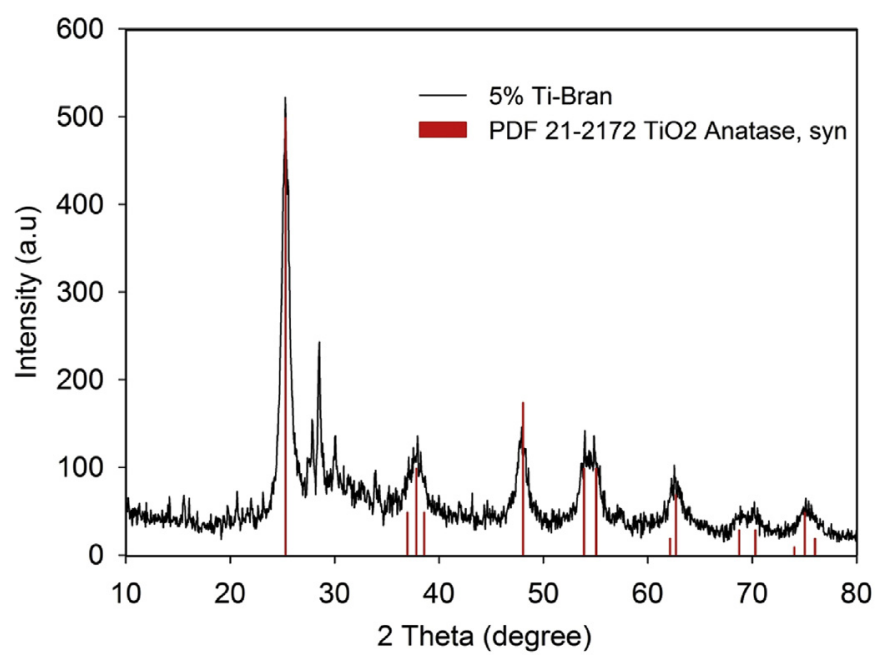

Fig. 1. XRD pattern of $5 \% \mathrm{Ti}$-Bran nanocomposite as compared to the diffraction lines of the pure anatase phase (red vertical lines). (For interpretation of the references to colour in this figure legend, the reader is referred to the web version of this article.)

incorporated $\mathrm{Ti}^{4+}$ species (Ti2p3/2, $458.3 \mathrm{eV}$ ) in the materials as clearly illustrated in Fig. 2. Interestingly, the fitting peak at ca. $454.5 \mathrm{eV}$ in the Ti2p spectra of $0.5 \%$ Ti-Bran catalyst might be ascribed to the substitution of oxygen by carbon species in the $\mathrm{TiO}_{2}$ lattice and formation of $\mathrm{Ti}-\mathrm{C}$ bond which can also be observed in the C1s spectra from the peak at $282.2 \mathrm{eV}$ (Liu et al., 2014; Sordello et al., 2014). The difference in the fitting peak for Ti2p of $454.5 \mathrm{eV}$ and $458.3 \mathrm{eV}$ is potentially resulted from the presence of $\mathrm{C}-\mathrm{Ti}-\mathrm{O}$ bond in the $\mathrm{TiO}_{2}$ lattice that the electronegativity of $\mathrm{C}(2.55)$ is lower than that of $\mathrm{O}(3.44)$ which results in more electron-rich in $\mathrm{Ti}-\mathrm{O}$ bond and further affects the difference in binding energy. This may also indicate the lattice distortion due to the presence of $\mathrm{C}$ atom (Neville et al., 2012), as similarly observed in the XPS spectra of other Ti-Bran catalysts. Ti content (Table 1) was also quantified via ICP/MS and was found to be in the range of $8-40 \mathrm{wt} \%$ of the final materials in the Ti-Bran samples after partial removal of the organics from wheat bran.

Nevertheless, nanoparticle sizes could remain relatively small probably due to the mechanochemical process between the titania precursor and the wheat bran as well as to the partial (not complete) elimination of part of the organic phase from wheat bran in the calcinations. Indeed, these results were in good agreement with TEM images of the synthesized Ti-Bran nanocomposites which clearly depicted the presence of highly dispersed and homogeneously distributed titania nanoparticles (black dots) on the remnants of the wheat bran feedstock (Fig. 3). The visualized nanoparticle sizes on TEM also correlated well with results of XRD patterns obtained using the Scherrer equation, with particle sizes around $10 \mathrm{~nm}$ (10\% Ti-Bran) and even smaller (5-7 nm) for lower

Table 1

Textural properties and Ti content of Ti-Bran nanocomposites.

\begin{tabular}{|c|c|c|c|c|c|}
\hline Sample & Surface area $\left(\mathrm{m}^{2} / \mathrm{g}\right)$ & Pore size $(\mathrm{nm})$ & Pore volume $\left(\mathrm{cm}^{3} / \mathrm{g}\right)$ & Ti content ${ }^{\mathrm{a}}(\mathrm{wt} \%)$ & Titania nanoparticle size ${ }^{\mathrm{b}}(\mathrm{nm})$ \\
\hline Bran & $<5$ & - & - & - & - \\
\hline $0.5 \%$ Ti-Bran & 10 & 27 & 0.07 & 8.1 & $4.6(5)$ \\
\hline 1\% Ti-Bran & 12 & 32 & 0.09 & 14.0 & $5.7(6)$ \\
\hline $2 \%$ Ti-Bran & 15 & 35 & 0.1 & 24.6 & $5.2(6)$ \\
\hline 5\% Ti-Bran & 13 & 25 & 0.1 & 36.5 & $6.2(7)$ \\
\hline $10 \%$ Ti-Bran & 20 & 30 & 0.1 & 41.4 & $7.8(10)$ \\
\hline
\end{tabular}

a Ti content measured by ICP/MS.

b Nanoparticle sizes was calculated using the Scherrer equation as compared to average particle size by TEM (in brackets), respectively. 

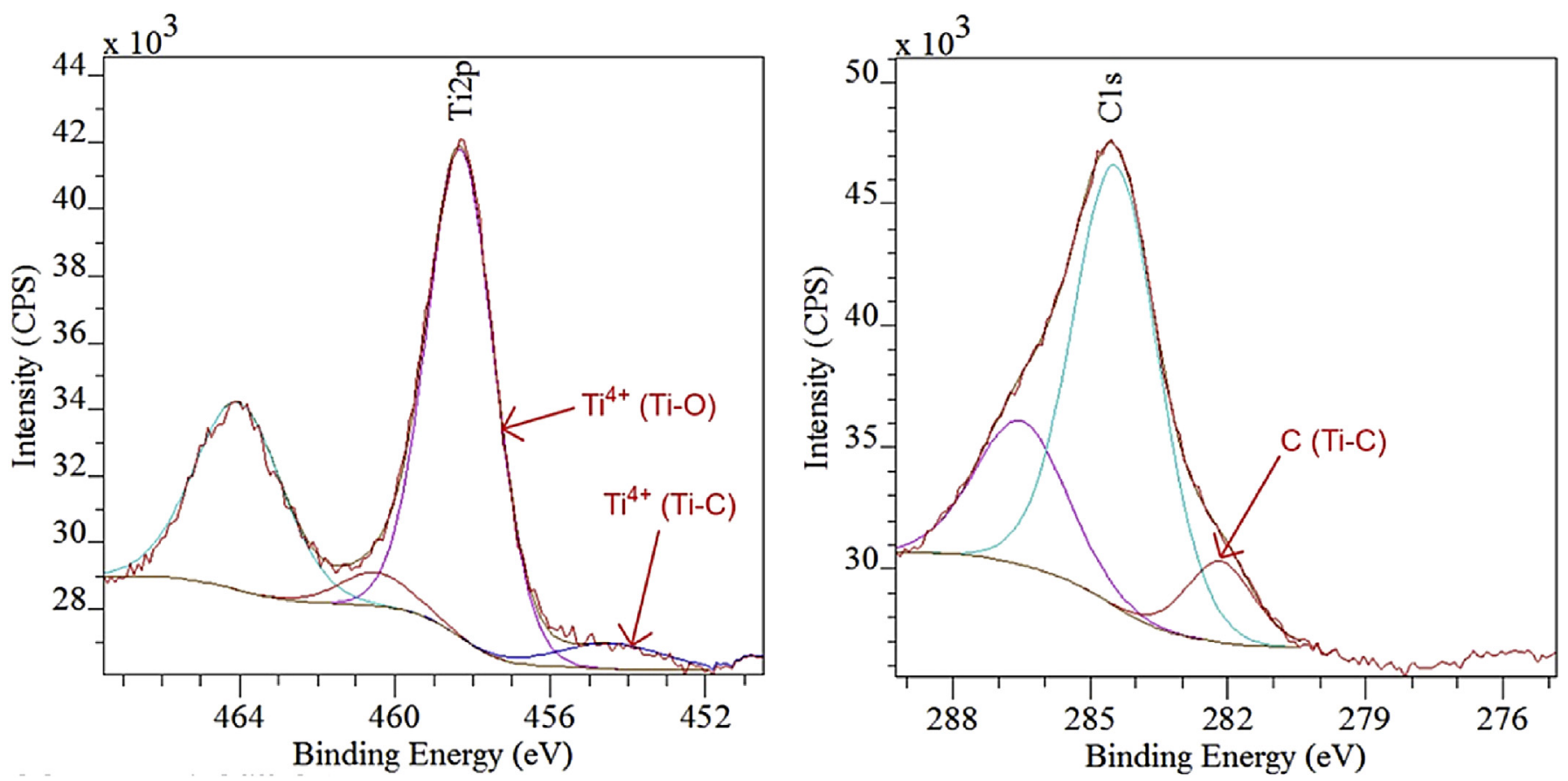

Fig. 2. XPS spectra of $0.5 \%$ Ti-Bran: Ti2p (left), C1s (right).
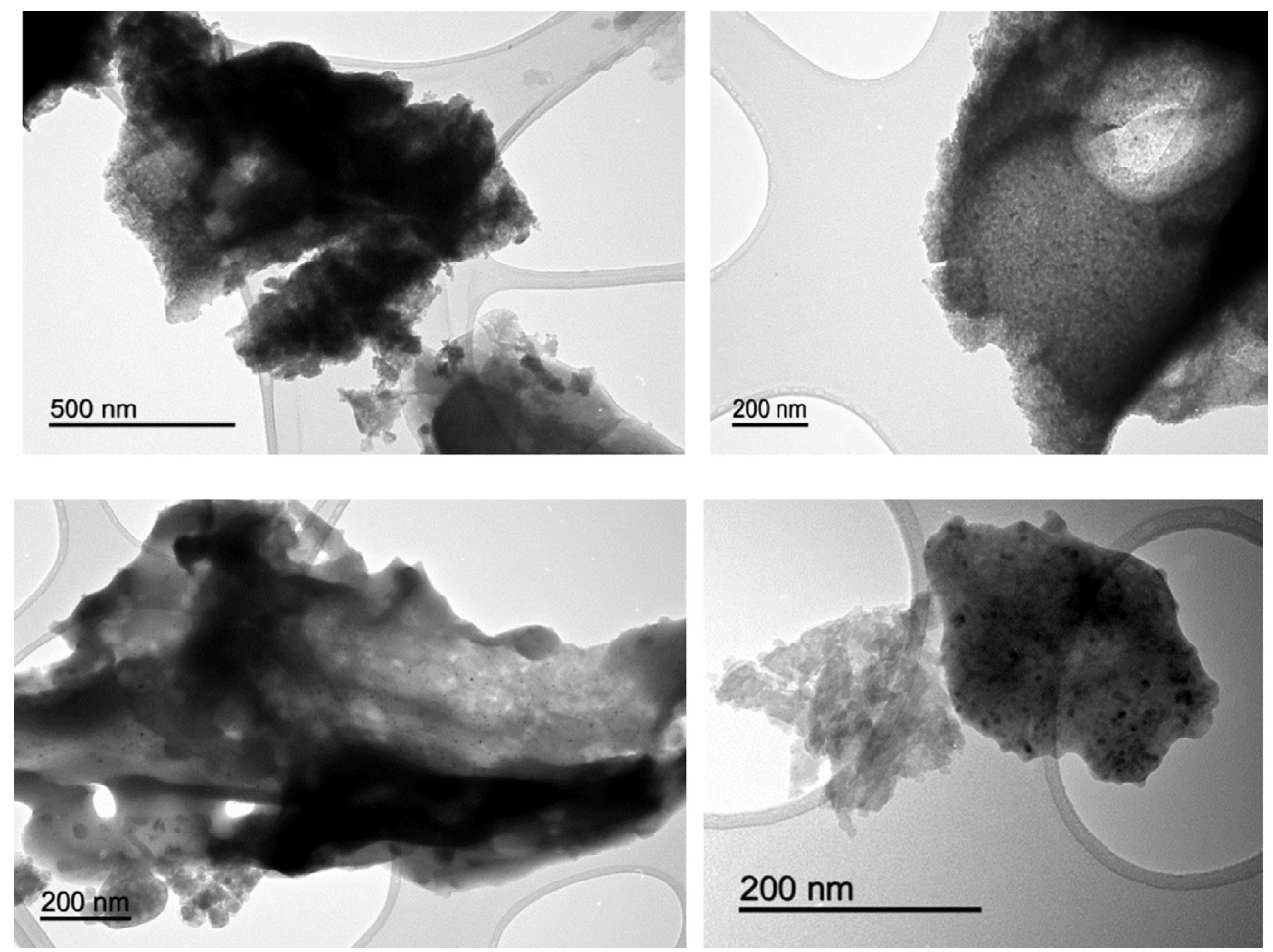

Fig. 3. TEM micrographs of $0.5 \%$ Ti-Bran (top images) and $10 \%$ Ti-Bran (bottom images). Titania nanoparticles could be clearly visualized as black dots.

loaded Ti-Bran nanocomposites (Fig. 3, top images). Some titania aggregates could also be observed in TEM images of the synthesized nanocomposites although these were clearly minor.

Optical absorption spectra of the synthesized photonanocomposites with different $\mathrm{TiO}_{2}$ content are shown in Fig. S2. As expected, the optical response of all the catalysts were extended into the visible light region with a slight enhancement of light absorption of all the samples achieved at a wavelength of around $400 \mathrm{~nm}$ as compared to that of pure anatase $\mathrm{TiO}_{2}(<387 \mathrm{~nm})$. The promotion of the light response could be attributed to the presence of carbon and trace elements which act as photosensitizer (Lettmann et al., 2001) and probably the newly created heterojunctions between $\mathrm{TiO}_{2}$ and the carbon support. The average band gap of the synthesized samples is ca. $3.0 \mathrm{eV}$, reduced as compared 
Table 2

Photocatalytic oxidation of benzyl alcohol using different photocatalysts.

\begin{tabular}{|c|c|c|c|c|}
\hline Catalyst & Conversion (\%) & Selectivity $^{\mathrm{a}} \mathrm{BHA}^{\mathrm{b}}(\%)$ & Yield BHA (\%) & Selectivity BAC ${ }^{\mathrm{C}}(\%)$ \\
\hline Photolysis & $<3$ & - & - & - \\
\hline $0.5 \%$ Ti-Bran & $<5$ & 73 & $<5$ & - \\
\hline 1\% Ti-Bran & 16 & 53 & 9 & $<5$ \\
\hline $2 \%$ Ti-Bran & 20 & 63 & 12 & $<5$ \\
\hline $5 \%$ Ti-Bran & 19 & 89 & 17 & $<5$ \\
\hline $10 \%$ Ti-Bran & 33 & 66 & 20 & $<5$ \\
\hline $\mathrm{TiO}_{2}(\mathrm{P}-25)$ & $>99$ & 32 & 33 & 6 \\
\hline
\end{tabular}

a Apart from BHA and BAC, the remaining selectivity to 100 corresponds mostly to $\mathrm{CO} 2$.

b BHA: benzaldehyde.

c BAC: benzoic acid.

to pure anatase $\mathrm{TiO}_{2}(3.2 \mathrm{eV})$. Details about the band gap energy and absorption threshold can be seen in Table S2.

After careful materials characterization, the application of the synthesized photonanocomposites was subsequently investigated in the selective photo-oxidation of benzyl alcohol to benzaldehyde. A summary of photocatalytic activity experiments has been presented in Table 2, while the comparison of photocatalytic performance in oxidation of benzyl alcohol between 10\% Ti-Bran and other reported $\mathrm{TiO}_{2}$ based catalysts was listed in Table S3. The conversion of the controlled photolysis (blank reaction, in the absence of catalysts) was found to be negligible $(<5 \%)$ after $4 \mathrm{~h}$ of illumination time, as well as the conversion with $0.5 \%$ Ti-Bran. Upon increasing Ti content, both conversion of starting material and yield of benzyl alcohol increased with the content of $\mathrm{TiO}_{2}$ on the carbonaceous nanocomposite. The maximum yield of benzaldehyde (ca. 20\% at 33\% conversion of benzyl alcohol) was achieved for $10 \%$ Ti-Bran catalyst with a selectivity of over $60 \%$. Comparatively, the P-25 Evonik titania photocatalyst provided a maximum of $33 \%$ yield, with quantitative conversion of starting material (mostly mineralization of benzyl alcohol takes place under the investigated conditions)(Colmenares et al., 2016). Taking into account the titania content in both materials, we can conclude that the designed titania-wheat bran nanocomposites outperform commercial titania nanomaterials in the investigated selective photo-oxidation. The higher selectivity towards benzaldehyde for Ti-Bran nanocomposites (see Table 2) may be attributed to the incorporation of carbon atoms on the $\mathrm{TiO}_{2}$ lattice. Additionally, researchers suggested that carbon acted as photosensitizer and the excited electrons could migrate to the conduction band of $\mathrm{TiO}_{2}$ which would be transferred to surface-absorbed $\mathrm{O}_{2}$ and forming superoxide anions. The superoxide anions could further convert to - $\mathrm{OH}$ and initiate the reaction (Yang et al., 2008). Over-oxidation products including benzoic acid were observed in the experiments using catalysts with $\mathrm{TiO}_{2}$ content $\geq 1.0 \%$, although these were obtained in relatively minor quantities.

\section{Conclusions}

This contribution was aimed to illustrate the potential of valorizing agroindustrial waste into a range of valuable materials, chemicals and fuels. The use of wheat bran, a residue with numerous available from the agricultural industry, as feedstock for the design of innovative photocatalytic nanocomposites for advanced applications in the selective conversion of benzyl alcohol to benzaldehyde, a well-known specialty chemical with important applications in the food, fragrances and cosmetics industries. The use of a simple, efficient and environmentally friendly technology (ball milling, mechanochemistry) provided the possibility to synthesizing titania-containing nanocomposites with promising textural and surface properties and useful photocatalytic activities in the selected photo-oxidation process. The optimum nanocomposite (10\% Ti-Bran) provided a $20 \%$ yield of benzaldehyde at $33 \%$ conversion of benzyl alcohol, comparable to that of P25 Evonik commercial titania utilized under the same conditions for comparative purposes.

\section{Acknowledgements}

Rafael Luque gratefully acknowledges Consejeria de Ciencia e Innovacion, Junta de Andalucia for funding project P10-FQM-6711. Funding from Marie Curie Actions under ITN Project Photo4Future (H2020-MSCA-ITN-2014-641861), especially for funding WO Ph.D studies. JCC, AMB and RL gratefully acknowledge support from COST Action FP1306 for networking and possibilities for meetings and future students exchange. Prof. Colmenares would like to thank the Institute of Physical Chemistry of the Polish Academy of Sciences for all support.

\section{Appendix A. Supplementary data}

Supplementary data related to this article can be found at http:// dx.doi.org/10.1016/j.jenvman.2016.07.013.

\section{References}

Adachi, K., Ohta, K., Mizuno, T., 1994. Photocatalytic reduction of carbon dioxide to hydrocarbon using copper-loaded titanium dioxide. Sol. Energy 53, 187-190. http://dx.doi.org/10.1016/0038-092X(94)90480-4.

Chang, J.T., Lai, Y.F., He, J.L., 2005. Photocatalytic performance of chromium or nitrogen doped arc ion plated-TiO2 films. Surf. Coat. Technol. 200, 1640-1644. http://dx.doi.org/10.1016/j.surfcoat.2005.08.118.

Chen, D., Jiang, Z., Geng, J., Wang, Q., Yang, D., 2007. Carbon and nitrogen co-doped $\mathrm{TiO}_{2}$ with enhanced visible-light photocatalytic activity. Ind. Eng. Chem. Res. 46, 2741-2746. http://dx.doi.org/10.1021/ie061491k.

Chen, D., Zhang, X., Lee, A.F., 2015. Synthetic strategies to nanostructured photocatalysts for $\mathrm{CO}_{2}$ reduction to solar fuels and chemicals. J. Mater. Chem. A 3, 14487-14516. http://dx.doi.org/10.1039/C5TA01592H.

Chen, X., Shen, S., Guo, L., Mao, S.S., 2010. Semiconductor-based photocatalytic hydrogen generation. Chem. Rev. 110, 6503-6570. http://dx.doi.org/10.1021/ cr1001645.

Colmenares, J.C., Luque, R., 2014. Heterogeneous photocatalytic nanomaterials: prospects and challenges in selective transformations of biomass-derived compounds. Chem. Soc. Rev. 43, 765-778. http://dx.doi.org/10.1039/ C3CS60262A.

Colmenares, J.C., Ouyang, W., Ojeda, M., Kuna, E., Chernyayeva, O., Lisovytskiy, D., De, S., Luque, R., Balu, A.M., 2016. Mild ultrasound-assisted synthesis of $\mathrm{TiO}_{2}$ supported on magnetic nanocomposites for selective photo-oxidation of benzyl alcohol. Appl. Catal. B Environ. 183, 107-112. http://dx.doi.org/10.1016/ j.apcatb.2015.10.034.

Francavilla, M., Pineda, A., Romero, A.A., Colmenares, J.C., Vargas, C. Monteleone, M., Luque, R., 2014. Efficient and simple reactive milling preparation of photocatalytically active porous $\mathrm{ZnO}$ nanostructures using biomass derived polysaccharides. Green Chem. 16, 2876-2885. http://dx.doi.org/ 10.1039/C3GC42554A.

Fujishima, A., Honda, K., 1972. Electrochemical photolysis of water at a semiconductor electrode. Nature 238, 37-38. http://dx.doi.org/10.1038/238037a0.

Herrmann, J.-M., Tahiri, H., Guillard, C., Pichat, P., 1999. Photocatalytic degradation of aqueous hydroxy-butandioic acid (malic acid) in contact with powdered and supported titania in water. Catal. Today 54, 131-141. http://dx.doi.org/10.1016/ S0920-5861(99)00175-3. 
Higashimoto, S., Kitao, N., Yoshida, N., Sakura, T., Azuma, M., Ohue, H., Sakata, Y., 2009. Selective photocatalytic oxidation of benzyl alcohol and its derivatives into corresponding aldehydes by molecular oxygen on titanium dioxide under visible light irradiation. J. Catal. 266, 279-285. http://dx.doi.org/10.1016/ j.jcat.2009.06.018.

Hoffmann, M.R., Hoffmann, M.R., Martin, S.T., Martin, S.T., Choi, W., Choi, W., Bahnemannt, D.W., Bahnemannt, D.W., 1995. Environmental applications of semiconductor photocatalysis. Chem. Rev. 95, 69-96. http://dx.doi.org/10.1021/ cr00033a004.

Hubert, C., Denicourt-Nowicki, A., Beaunier, P., Roucoux, A., 2010. $\mathrm{TiO}_{2}$-supported $\mathrm{Rh}$ nanoparticles: from green catalyst preparation to application in arene hydrogenation in neat water. Green Chem. 12, 1167. http://dx.doi.org/10.1039/ c004079g.

Irie, H., Watanabe, Y., Hashimoto, K., 2003. Nitrogen-concentration dependence on photocatalytic activity of $\mathrm{TiO}_{2}$-xNx powders. J. Phys. Chem. B 107, 5483-5486. http://dx.doi.org/10.1021/jp030133h.

Lettmann, C., Hildenbrand, K., Kisch, H., Macyk, W., Maier, W.F., 2001. Visible light photodegradation of 4-chlorophenol with a coke-containing titanium dioxide photocatalyst. Appl. Catal. B Environ. 32, 215-227. http://dx.doi.org/10.1016/ S0926-3373(01)00141-2.

Li, C.-J., Xu, G.-R., Zhang, B., Gong, J.R., 2012. High selectivity in visible-light-driven partial photocatalytic oxidation of benzyl alcohol into benzaldehyde over single-crystalline rutile $\mathrm{TiO}_{2}$ nanorods. Appl. Catal. B Environ. 115, 201-208. http://dx.doi.org/10.1016/j.apcatb.2011.12.003.

Linsebigler, A.L., Linsebigler, A.L., Yates Jr., J.T., Lu, G., Lu, G., Yates, J.T., 1995. Photocatalysis on $\mathrm{TiO}_{2}$ surfaces: principles, mechanisms, and selected results. Chem. Rev. 95, 735-758. http://dx.doi.org/10.1021/cr00035a013.

Liu, J., Zhang, Q., Yang, J., Ma, H., Tade, M.O., Wang, S., Liu, J., 2014. Facile synthesis of carbon-doped mesoporous anatase $\mathrm{TiO}_{2}$ for the enhanced visible-light driven photocatalysis. Chem. Commun. 50, 13971-13974. http://dx.doi.org/10.1039/ C4CC05544F.

Marotta, R., Di Somma, I., Spasiano, D., Andreozzi, R., Caprio, V., 2011. Selective oxidation of benzyl alcohol to benzaldehyde in water by $\mathrm{TiO}_{2} / \mathrm{Cu}(\mathrm{II}) / \mathrm{UV}$ solar system. Chem. Eng. J. 172, 243-249. http://dx.doi.org/10.1016/j.cej2011.05.097.

Neville, E.M., Mattle, M.J., Loughrey, D., Rajesh, B., Rahman, M., MacElroy, J.M.D., Sullivan, J.A., Thampi, K.R., 2012. Carbon-doped $\mathrm{TiO}_{2}$ and carbon, tungstencodoped $\mathrm{TiO}_{2}$ through sol-gel processes in the presence of melamine borate: reflections through photocatalysis. J. Phys. Chem. C 116, 16511-16521. http:// dx.doi.org/10.1021/jp303645p.

Ni, M., Leung, M.K.H., Leung, D.Y.C., Sumathy, K., 2007. A review and recent developments in photocatalytic water-splitting using $\mathrm{TiO}_{2}$ for hydrogen production. Renew. Sustain. Energy Rev. 11, 401-425. http://dx.doi.org/10.1016 | j.rser.2005.01.009.

Ohno, T., Akiyoshi, M., Umebayashi, T., Asai, K., Mitsui, T., Matsumura, M., 2004 Preparation of S-doped $\mathrm{TiO}_{2}$ photocatalysts and their photocatalytic activities under visible light. Appl. Catal. A Gen. 265, 115-121. http://dx.doi.org/10.1016 j.apcata.2004.01.007.

Ohno, T., Mitsui, T., Matsumura, M., 2003a. Photocatalytic activity of S-doped $\mathrm{TiO}_{2}$ photocatalyst under visible light. Chem. Lett. 32, 364-365. http://dx.doi.org/ 10.1246/cl.2003.364.

Ohno, T., Tokieda, K., Higashida, S., Matsumura, M., 2003b. Synergism between rutile and anatase $\mathrm{TiO}_{2}$ particles in photocatalytic oxidation of naphthalene. Appl. Catal. A Gen. 244, 383-391. http://dx.doi.org/10.1016/S0926-860X(02) 00610-5.

Shen, Y., Xiong, T., Li, T., Yang, K., 2008. Tungsten and nitrogen co-doped $\mathrm{TiO}_{2}$ nanopowders with strong visible light response. Appl. Catal. B Environ. 83, 177-185. http://dx.doi.org/10.1016/j.apcatb.2008.01.037.

Sordello, F., Zeb, G., Hu, K., Calza, P., Minero, C., Szkopek, T., Cerruti, M., 2014. Tuning $\mathrm{TiO}_{2}$ nanoparticle morphology in graphene- $-\mathrm{TiO}_{2}$ hybrids by graphene surface modification. Nanoscale 6, 6710. http://dx.doi.org/10.1039/c4nr01322k.

Spasiano, D., del Pilar Prieto Rodriguez, L., Olleros, J.C., Malato, S., Marotta, R. Andreozzi, R., 2013. $\mathrm{TiO}_{2} / \mathrm{Cu}$ (II) photocatalytic production of benzaldehyde from benzyl alcohol in solar pilot plant reactor. Appl. Catal. B Environ. 136-137, 56-63. http://dx.doi.org/10.1016/j.apcatb.2013.01.055.

Sun, J., Qiao, L., Sun, S., Wang, G., 2008. Photocatalytic degradation of Orange G on nitrogen-doped $\mathrm{TiO}_{2}$ catalysts under visible light and sunlight irradiation. J. Hazard. Mater 155, 312-319. http://dx.doi.org/10.1016/j.jhazmat.2007.11.062.

Virkutyte, J., Baruwati, B., Varma, R.S., 2010. Visible light induced photobleaching of methylene blue over melamine-doped $\mathrm{TiO}_{2}$ nanocatalyst. Nanoscale 2, 1109-1111. http://dx.doi.org/10.1039/c0nr00089b.

Virkutyte, J., Varma, R.S., 2010. Fabrication and visible light photocatalytic activity of a novel $\mathrm{Ag} / \mathrm{TiO}_{2}-\mathrm{xNx}$ nanocatalyst. New J. Chem. 34, 1094-1096. http:// dx.doi.org/10.1039/c0nj00268b.

Wu, G., Nishikawa, T., Ohtani, B., Chen, A., 2007. Synthesis and characterization of carbon-doped $\mathrm{TiO}_{2}$ nanostructures with enhanced visible light response. Chem. Mater 19, 4530-4537. http://dx.doi.org/10.1021/cm071244m.

Yang, X., Cao, C., Erickson, L., Hohn, K., Maghirang, R., Klabunde, K., 2008. Synthesis of visible-light-active $\mathrm{TiO}_{2}$-based photocatalysts by carbon and nitrogen doping. J. Catal. 260, 128-133. http://dx.doi.org/10.1016/j.jcat.2008.09.016.

Yu, J., Low, J., Xiao, W., Zhou, P., Jaroniec, M., 2014. Enhanced photocatalytic $\mathrm{CO}_{2}$ reduction activity of anatase $\mathrm{TiO}_{2}$ by coexposed $\{001\}$ and $\{101\}$ facets. J. Am. Chem. Soc. 136, 8839-8842. http://dx.doi.org/10.1021/ja5044787. 